\title{
Evaluation of Urban Spatial Equality Based on Accessibility to Economic Activities: Beijing as a Case Study
}

\author{
Xinyu Yang, ${ }^{1,2}$ Fangqu Niu $\mathbb{D}^{1,},{ }^{1,2,3}$ and Dongqi Sun $\mathbb{D i D}^{1,2}$ \\ ${ }^{1}$ Key Laboratory of Regional Sustainable Development Modeling, \\ Institute of Geographic Sciences and Natural Resources Research, Chinese Academy of Sciences, Beijing 100101, China \\ ${ }^{2}$ College of Resources and Environment, University of Chinese Academy of Sciences, Beijing 100049, China \\ ${ }^{3}$ Collaborative Innovation Center for Geopolitical Setting of Southwest China and Borderland Development, \\ Kunming 650500, China
}

Correspondence should be addressed to Fangqu Niu; niufq@lreis.ac.cn and Dongqi Sun; sundq@igsnrr.ac.cn

Received 4 July 2020; Revised 15 August 2020; Accepted 30 August 2020; Published 8 October 2020

Academic Editor: Jun Yang

Copyright (c) 2020 Xinyu Yang et al. This is an open access article distributed under the Creative Commons Attribution License, which permits unrestricted use, distribution, and reproduction in any medium, provided the original work is properly cited.

Urban space is the spatial projection of various social and economic activities. Given the complexity of urban functions and the ongoing expansion of urban areas, the spatial differentiation of various economic activities within cities tends to become more and more clear; moreover, there tends to be spatial inequalities in resource allocation. Taking Beijing as an example, this study develops a spatial accessibility model at the town (jiedao) level by integrating the spatial distribution of economic activities with the transport system and evaluating the accessibility (at the spatial level) to various economic activities. The equality of the residents' economic-related travel activity is also evaluated in line with the population distribution. The results show that the accessibility to economic activities generally decreases in going from the urban center to the peripheral suburbs, and this "core to edge" difference is readily apparent. In general, residents tend to choose areas to settle in which have a high degree of accessibility, however, the attractiveness of accessibility is constrained within certain limits, as evidenced by a reduction of population in the center of Beijing. Additionally, there are inequalities with respect to traveling experiences. For instance, $27.8 \%$ of residents experience very convenient travel conditions, mostly in the Xicheng, Dongcheng, and Haidian districts, and this equates to a high level of accessibility; about half of the residents in Beijing live in areas ranked as being of medium accessibility and where the distribution of economic activities and transportation facilities are insufficient. Residents living in the outlying Changping, Mentougou, Shunyi, and Fangshan districts have relatively poor access to transportation indicating a low level of accessibility. Overall, the evaluation method for spatial equality considers comprehensively the distribution of economic activities, transportation, and population distribution and can provide a reference framework for optimization of the urban spatial structure to improve urban spatial equality.

\section{Introduction}

The city is the place where residents engage in daily activities, and the urban space is the spatial projection of various social and economic activities in a specific urban area, which embody the manifestation and spatiotemporal characteristics of all economic activities $[1,2]$. Since the 1970s, given the complexity of urban functions and the ongoing expansion of urban areas, the differentiation of various economic activities at the regional level within the city has become more and more evident. In developing countries where there may be rapid growth rates in cities, it is common to have conflict between the supply and service levels of economic activities [3]. In general, there is a lack of service location capability in such cities due to an inequitable allocation of resource space.

Spatial equality can be viewed as the spatial distribution of economic activity opportunities that can serve the needs and preferences of all individuals on an equitable basis [4]. One of the main objectives of research on spatial equality is to determine whether the distribution of urban services is fair and can adapt to the spatial patterns of society and the economy [5]. As the process of urbanization continues to 
accelerate, the populations in urban centers are increasing rapidly, and the nonadaptability between the support facilities underpinning urban economic activity and the needs of residents has become more and more critical. Considering the differences in economic activity-related journeys made by residents, spatial equality, in the context of transport accessibility, can be better understood in terms of people having different attributes and requirements for undertaking such journeys. Regarding research on spatial equality, there has been an abundance of data from independent studies. For example, Talen et al. used data from Pueblo, Colorado and Macon, Georgia (USA) to directly link the spatial distribution of park accessibility with the spatial distribution of socioeconomic factors of the population; in so doing the researchers compared the differences in fairness of park distribution in the two cities and proposed a new method for fairness evaluation [6]. Chen et al. selected the ancient city of Shaoxing, Zhejiang province (China) as an example and used the spatial distribution of residential buildings to estimate the spatial distribution of permanent residents in the region to calculate, at a fine scale, the equality of citizens' access to green park space [7]. It was demonstrated there were two main factors affecting the evaluation of the spatial equality of urban economic activity: one was the accessibility distribution characteristics of economic activity; the other was the spatial distribution associated with the travel needs of the residents. From a spatial perspective, on the basis of considering the accessibility of various economic activities, further attention should be given to the equality of the residents' journey opportunities, a factor which is of great significance for improving the urban spatial structure, optimizing the allocation of urban resources, and further promoting social equality.

The concept of accessibility was originally derived from the concept of transportation geography and later expanded to include the study of urban planning, economic geography, sociology, and other disciplines [8]. This concept was first proposed by Hansen in the 1950s and defined as the degree of interconnection for a given point with all other points on the same surface [9]. Since then, multiple definitions of accessibility have been proposed for tackling different research problems $[10,11]$. The spatial accessibility of economic activities mainly refers to the convenience for people that their commuting and daily traveling needs between a given location and a target location are met by a certain mode of transport [12]. Spatial accessibility reflects the fairness of access to specific economic activities by the inhabitants of different areas and, accordingly, spatial accessibility studies may identify areas where such facilities are lacking. Accessibility is not only affected by geographical or spatial distance but also by economic, informational, and behavioral influences [13]. After the midtwentieth century, accessibility gradually became a reference for spatial planning of urban economic activities, and the study objects cover almost all categories of economic activity, including green space $[6,14]$, medical service facilities [15-18], educational facilities [19-21], and other services. The research methods employed in spatial planning and accessibility include the buffer analysis method, the cost-weighted distance method, the two-step floating catchment area method, and the gravity model method. Different methods have their own advantages and disadvantages; moreover, they have different requirements for data and reflect different aspects of accessibility. To date, no method is able to cover all aspects of accessibility [22, 23].

There is a close connection between spatial equality and accessibility. From the perspective of supply and demand, spatial equality emphasizes the differences of economic activities acquired by different social groups or different regions, among which the distribution characteristics of accessibility is one of the important factors affecting space equality [24]. Equality is an extension of the concept of accessibility [25], and accessibility provides an effective quantitative approach for the study of spatial equality. In the evaluation of equality, various statistical indicators that can detect imbalances and gaps are often used to characterize the balance of regional resource allocation, examples of such include the coefficient of variation (CV) [26], the Gini coefficient [27], and the Theil index $[28,29]$. However, these indicators only discuss the balance of the distribution from the perspective of the number of economic activity opportunities and ignore the differences in the spatial externalities of economic activities, the transportation networks, and the spatial scales of the research; thus, they cannot truly reflect the distribution of economic activity opportunities in an urban setting. Different from sociology, which is concerned with the allocation and deprivation level of social resources from the perspective of constructing social equality [30], geography is concerned with measuring the location equality and the spatial deprivation from the perspective of space; the accessibility analysis combined with GIS technology is the main method used to measure the spatial equality of urban economic activities. Based on the relevant literature, the study of urban spatial accessibility and equality has resulted in a substantial body of research data and information; however, there are some aspects of the traditional evaluation methods that need to be improved as a matter of urgency. First, most studies focus on a single measure of accessibility for an economic activity-related journey [31], while urban economic activities are highly diversified. The evaluation results based on a single economic activity are onesided. Second, many researchers use the spatial distribution of accessibility to explain the spatial equity of economic activities $[32,33]$, ignoring the needs of the participants engaged in economic activities, namely, the equality of journey opportunities for residents undertaking the economic activities, so the approach cannot measure well the equality of resource allocation. Third, many studies focus on large-scale urban intercity spatial equality $[34,35]$, while microanalysis of a city's internal spatial equality is weak, and less attention is paid to the difference in location equality at the town (jiedao) scale. Therefore, it is necessary to move beyond the limitation of traditional spatial accessibility and consider comprehensively the distribution of opportunities for various economic activities, the transportation costs, and the population distribution from a more detailed scale perspective in order to measure the equality of the residents' economic activity-related journeys.

This study, in taking Beijing as an example, integrates the distribution of various economic activities and the 
transportation system and establishes a spatial accessibility model at the town scale to evaluate the convenience with respect to various economic activities of traveling to the city center from each start location. On this basis, combined with the data for the spatial distribution of the residents, the equality of economic activity-related journey opportunities for the residents in the study area may be evaluated and further explored. Such work provides a reference framework for optimizing the urban spatial structure and improving urban spatial equality.

\section{Data and Methodology}

2.1. Study Area and Data. Beijing, the capital of China, has a clear single-center urban structure and is commonly classified into three subregions: the inner city, the inner-ring suburbs, and the outer-ring suburbs. The study area, the main metropolitan area of Beijing, consists of 12 urban districts and 239 towns (Dongcheng, Xicheng, Chaoyang, Haidian, Fengtai, Shijingshan, Daxing, Tongzhou, Shunyi, Changping, Mentougou, and Fangshan). Shaped by history and government policies, the study area has a large population (approximately 21 million) and a high concentration of diverse economic activities. In 2018, permanent residents accounted for $92.1 \%$ of the city's total population, and there was a high degree of economic activity and workforce mobility in the city. This study takes the town (jiedao) as the study unit. The town is the basic administrative unit for cities in China [36] and also the basic geographic unit for people when assessing various economic activities (the town-based unit has been shown to reflect more spatial heterogeneity than a county-based unit). Figure 1 shows the study area at the district and town levels.

The spatial data used in the study included Beijing's traffic network data (including highways, urban expressways, national highways, provincial roads, county roads, and subway lines), which were obtained from the open street map (OSM) (http://www.openstreetmap.org/\#map=11/30. 1876/114.9417) and the economic activity distribution data and the population data at the town scale for the sixth National Census in 2010. Among them, Beijing's economic activity distribution data are from the economic census data combined with field survey data. After obtaining the relevant information for each town unit, the API (Application Programming Interface), provided by the electronic map vendor, was matched with the POI (Point of Interest) to determine the spatial coordinates of each enterprise. A total of more than 700,000 enterprise information data points were obtained, including the enterprise's spatial location, asset scale, and the number of employees. The data cover almost all companies, schools, research institutes, hospitals, and other work units in Beijing. Based on these data and using GIS spatial analysis, detailed spatial distribution maps for various economic activities in Beijing were produced.

2.2. Methodology. In this paper, "spatial accessibility" of urban economic activities is defined as the convenience of reaching the target economic activity by a certain mode of

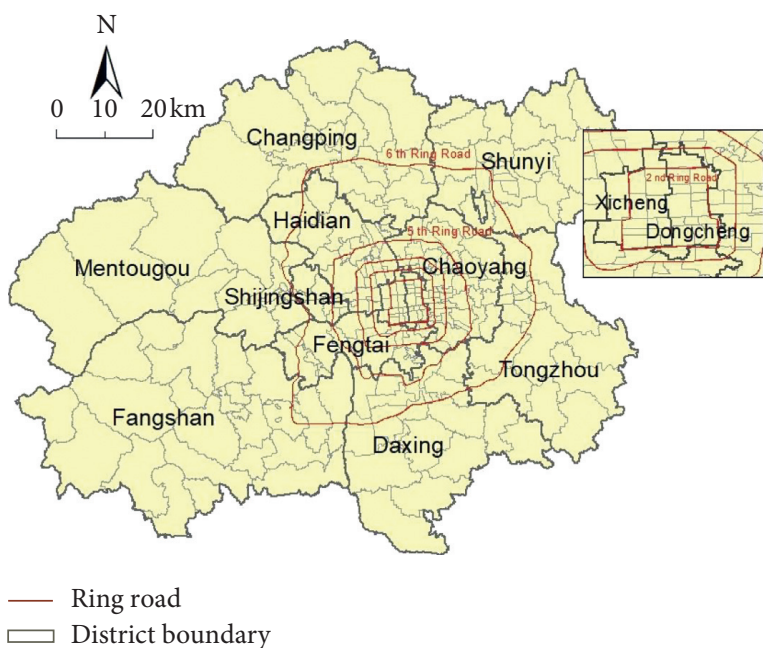

FIgURE 1: Administrative divisions for the study area (Beijing).

transport starting out from a certain town. Four representative categories of economic activity in urban life, namely, employment, education, medical care, and shopping and leisure, were selected to calculate the respective spatial accessibilities. Taking employment as an example, the spatial accessibility of town $i$ refers to the convenience of reaching all the employment positions in the city from town $i$, which represents the location conditions of that town. There are two main factors which influence the outcome: one is the distribution of all kinds of economic activity in the city; the other is the transport conditions associated with attaining all these types of economic activity [37]. Among them, the traffic conditions represent the convenience of moving between towns from the physical traffic level point of view. Generally, the routes are characterized by the shortest distance, the minimum time cost, or the economic cost. We can also consider comprehensively the time and economic costs associated with the routes, which we call the comprehensive cost.

Based on the road traffic road network (consisting of various levels of traffic routes), this paper uses the shortest path GIS algorithm to identify the shortest path between towns and taking the shortest time as the index. The result of the traffic evaluation is an $m \times m$ matrix, which represents the shortest journey time between two towns, where $m$ is the number of towns in the urban space. Based on the above considerations, the formula for the model is

$$
a_{i}=-\frac{1}{\lambda}\left\{\ln \sum_{j} W_{j} \exp \left(-\lambda d_{i j}\right)\right\},
$$

where $a_{i}$ is the spatial accessibility of urban town $i, W_{j}$ is the number of economic activity opportunities in the town $j$, $\sum_{j} W_{j}$ is the sum of all the economic activities in the city, $d_{i j}$ is the transportation cost from $i$ to $j$, and the distribution coefficient $d_{i j}$ represents the sensitivity of different traffic modes to distance changes. This paper does not delineate the transportation mode, so the value of $\lambda$ can be 1 . When calculating the spatial accessibility of town $i$, the distribution 
of economic activity opportunities in all towns of the city will be summed.

The coefficient of the distance parameter $d_{i j}$ in the model is negative $(-\lambda)$. When the distance increases, the value of the exponential function $(\exp ())$ becomes smaller, and the weight $W_{j}$ of town $j$ is reduced. Therefore, if it is difficult for a person in town $i$ to reach town $j$ (the value of $d_{i j}$ is very high), hence the opportunities in $j$ will become meaningless; conversely, if $j$ can be easily reached (the value of $d_{i j}$ is small), the number of opportunities has a great impact. Given that the evaluation result for the dimension of the above model is the same as the dimension of the transportation cost $(d)$, the larger the value, the smaller the accessibility. Therefore, formula (1) is further processed. As shown in formula (2), where $A_{i}$ is the accessibility of the town $i$ after normalization, the higher the value of $A_{i}$, the higher the accessibility:

$$
A_{i}=\max _{1 \leq i \leq n}\left\{a_{i}\right\}-a_{i} .
$$

The reason for using the above logarithmic summation to evaluate accessibility is that the model includes two aspects, that is, the transportation system and the economic activity distribution; the model evaluates accessibility based on the city's distribution of economic activities, without setting a threshold. Formula (1) is based on previous research and is an extension of McFadden's [38] stochastic utility theory.

\section{Spatial Analysis of Accessibility with Respect to Economic Activity}

3.1. Accessibility by Type of Economic Activity. The opportunities for residents to benefit from the diverse types of resources due to economic activity are determined by their location. According to formulae (1) and (2), to calculate the accessibility to various economic activities, it is necessary to set the weight for each town, that is, to set the number of activity opportunities $W_{j}$. To avoid errors caused by the sizes of different enterprises, this paper adopts the number of employees as a measure of the distribution of opportunities for economic activities. Accordingly, employment opportunities are the total number of jobs in each town, and the number of opportunities for accessing education, medical care, shopping, and leisure activities is given by the number of employees in the medical, educational, and other service industries in each town. The evaluation results for accessibility with respect to the various economic activities are presented in Figure 2.

It can be seen that the spatial patterns of accessibility for the different types of economic activities in Beijing are quite similar with a general decrease occurring in going from the city center to the peripheral suburbs, and the "core to edge" difference in spatial accessibility is clear. That is, the patterns of economic activity reflect the fact that the distribution densities of the activities gradually decrease in going from the central urban area to the peripheral suburbs. In terms of employment accessibility (Figure 2(a)), locations within the $4^{\text {th }}$ Ring Road have a strong geographical advantage. This is because most of the employment opportunities in Beijing are distributed in the main urban areas within the $4^{\text {th }}$ Ring Road, and the transportation facilities there are conducive to commuting. As far as educational journeys are concerned (Figure 2(b)), the western region within the $5^{\text {th }}$ Ring Road has clear advantages, and the suburbs between the $4^{\text {th }}$ Ring Road and the $5^{\text {th }}$ Ring Road also have a high degree of accessibility. This reflects the fact that these areas have a large number of high-quality primary and secondary schools and colleges; hence, the distribution of educational resources is relatively dense. Medical accessibility (Figure 2(c)) and shopping and leisure accessibility (Figure 2(d)) generally have similar distributions and the advantageous locations are concentrated in the main urban areas within the 5th Ring Road. This is due to the development in the past of a "cake spreading" effect for various medical facilities, large shopping malls, and supermarkets with clusters forming in this urban area. Given the current deteriorating traffic situation in Beijing, relocation of medical activities, shopping, leisure, and other economic activities is imperative.

The top and bottom ten towns for the various economic activities are presented in Table 1. Among them, seven of the top ten towns for employment accessibility are located in Xicheng District, including the top three towns with the highest accessibility, that is, Jinrongjie, Yuetan, and Xinjiekou. This result illustrates that Xicheng District has good employment accessibility, benefits from convenient transportation conditions, and a high concentration of diverse employment units. The next ranked districts for employment accessibility are Haidian and Dongcheng, where there are only two towns and one town, respectively, among the top ten; moreover, employment accessibility is significantly weaker than in Xicheng District. Among the ten lowestranking towns for employment accessibility, seven are located in Fangshan District, including the town of Puwa which is the lowest ranked.

Among the top ten towns for educational accessibility, five are located in Xicheng District and four are located in Haidian District. Xinjiekou, Huayuanlu, and Jinrongjie rank among the top three towns. This is due to a large number of high-quality primary and secondary schools and colleges located in the Haidian and Xicheng districts, where there are ample educational resources and well-developed transportation facilities. In addition, Chaoyang District ranks seventh in terms of education accessibility. However, Dongcheng District, which is also located in the core urban area, is not in the top ten, indicating that access to educational resources is relatively poor. The bottom ten towns with respect to educational accessibility are all located in the Fangshan and Mentougou districts.

Seven of the top ten towns for medical accessibility are located in Xicheng District, including the top three towns with the highest accessibility, that is, Xinjiekou, Jinrongjie, and Yuetan. This situation reflects the fact that Xicheng District is endowed with substantial medical resources and has significant advantages with respect to travel accessibility. In addition, the districts of Haidian, Dongcheng, and Chaoyang each have only one town in the top ten; thus, medical accessibility needs to be further strengthened in 


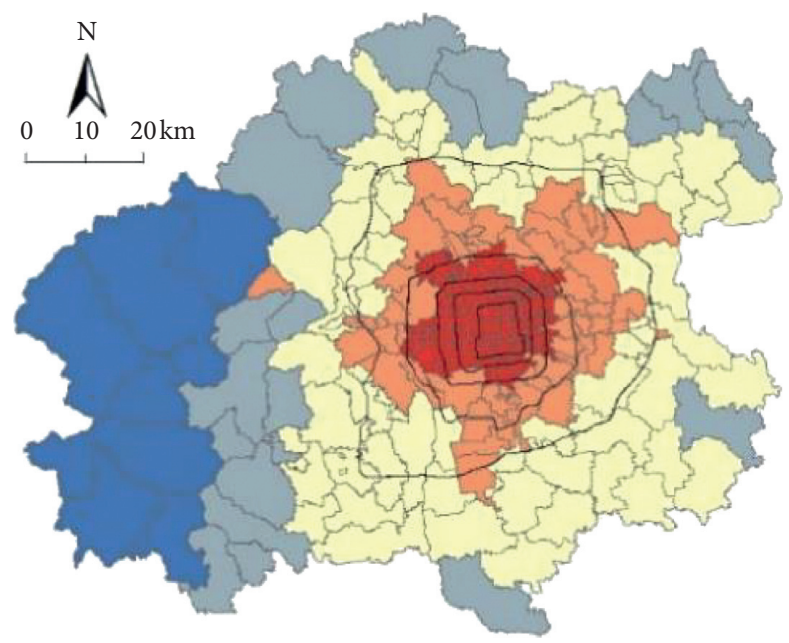

_ Ring road

Employment accessibility

$$
\begin{array}{ll}
\square 0.00-47.67 & \square 112.93-126.30 \\
\square \text { 47.68-92.07 } & \square 126.31-138.32 \\
\square 92.08-112.92 &
\end{array}
$$

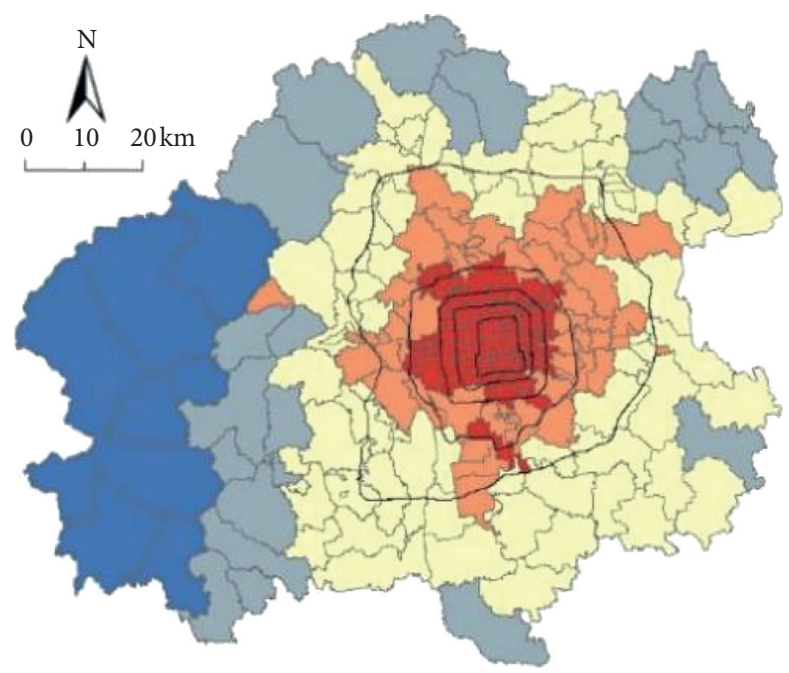

— Ring road

Medical accessibility

$\square 0.00-47.88$

$\square$ 47.89-92.49

$\square 92.50-112.23$ (a)

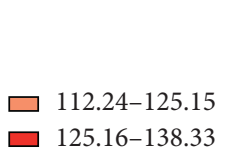

(c)

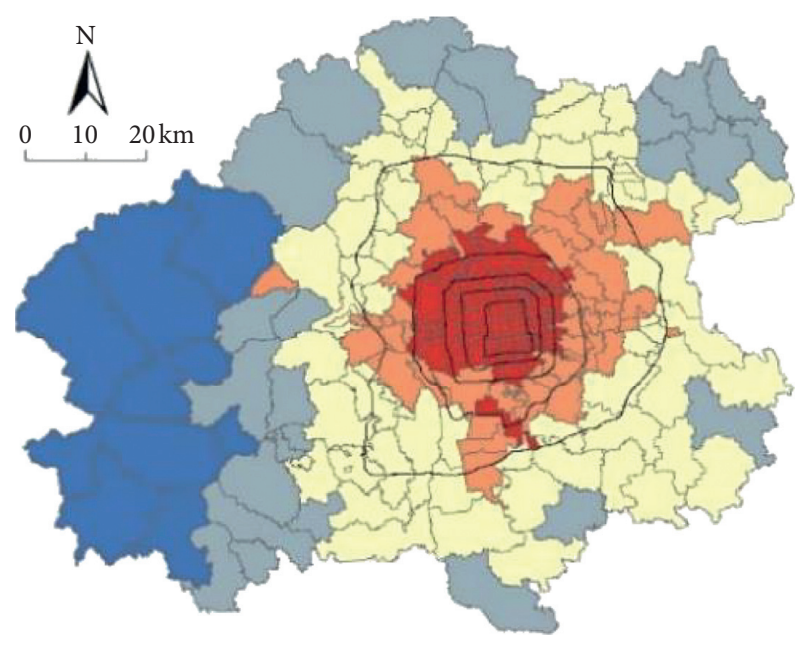

\section{— Ring road}

Educational accessibility
$\square 0.00-57.73$
$\square$ 57.74-94.36
$\square 94.37-112.14$
$\square$ 112.15-124.53

(b)

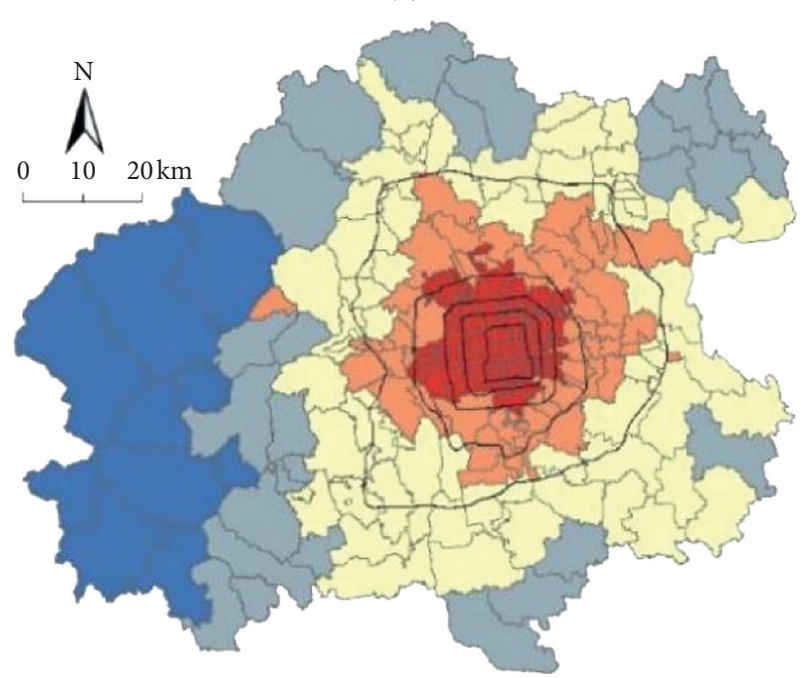

— Ring road

Shopping and leisure accessibility

$\square 0.00-57.90$

$\square 112.25-124.20$

$\square$ 57.91-95.05

$\square 95.06-112.24$ $\square$ 124.21-135.41

(d)

Figure 2: Distribution maps for accessibility by type of economic activity. (a) Employment accessibility. (b) Educational accessibility. (c) Medical accessibility. (d) Shopping and leisure accessibility.

these districts. The lowest ten towns for medical accessibility are all located in the Fangshan and Mentougou districts.

In terms of shopping and leisure accessibility, Xicheng District has a significant geographical advantage with eight towns being ranked in the top ten. Among them, Yuetan, Jinrongjie, and Xinjiekou are in the top three. In other areas, only one town in each of Ganjiakou in Haidian District and Donghuamen in Dongcheng District rank in the top ten. This indicates that shopping and leisure facilities are highly concentrated in Xicheng District, while shopping and leisure facilities in other areas are less than adequate. The lowest ranked ten towns in terms of shopping and leisure accessibility are all located in the Fangshan and Mentougou districts.

In general, the towns with high accessibility to diverse economic activities are mostly in Xicheng District, of which Jinrongjie, Yuetan, Xinjiekou, and Shichahai are among the top ten towns. The distribution of the low 
TABLE 1: The top and bottom ten towns for accessibility to different economic activities.

\begin{tabular}{|c|c|c|c|c|c|c|c|c|}
\hline & \multicolumn{2}{|c|}{ Employment accessibility } & \multicolumn{2}{|c|}{ Educational accessibility } & \multicolumn{2}{|c|}{ Medical accessibility } & \multicolumn{2}{|c|}{$\begin{array}{l}\text { Shopping and leisure } \\
\text { accessibility }\end{array}$} \\
\hline & Jiedao (town) & District & Jiedao (town) & District & Jiedao (town) & District & Jiedao (town) & District \\
\hline 1 & Jinrongjie & Xicheng & Xinjiekou & Xicheng & Xinjiekou & Xicheng & Yuetan & Xicheng \\
\hline 2 & Yuetan & Xicheng & Huayuanlu & Haidian & Jinrongjie & Xicheng & Jinrongjie & Xicheng \\
\hline 3 & Xinjiekou & Xicheng & Jinrongjie & Xicheng & Yuetan & Xicheng & Xinjiekou & Xicheng \\
\hline 4 & Xichang'anjie & Xicheng & Desheng & Xicheng & Shichahai & Xicheng & Ganjiakou & Haidian \\
\hline 5 & Ganjiakou & Haidian & Ganjiakou & Haidian & Ganjiakou & Haidian & Xichang'anjie & Xicheng \\
\hline 6 & Shichahai & Xicheng & Yuetan & Xicheng & Xichang'anjie & Xicheng & Zhanlanlu & Xicheng \\
\hline 7 & Guang'anmennei & Xicheng & Anzhen & Chaoyang & Zhanlanlu & Xicheng & Guang'anmennei & Xicheng \\
\hline 8 & Zhanlanlu & Xicheng & Beitaipingzhuang & Haidian & Donghuamen & Dongcheng & Shichahai & Xicheng \\
\hline 9 & Donghuamen & Dongcheng & Shichahai & Xicheng & Guang'anmennei & Xicheng & Donghuamen & Dongcheng \\
\hline 10 & Yangfangdian & Haidian & Zhongguancun & Haidian & Anzhen & Chaoyang & Niujie & Xicheng \\
\hline 230 & & & & & & & & han \\
\hline 231 & Zhangfang & han & Zhan & $\tan$ & Zhan & & Zhar & han \\
\hline 232 & Da'anshan & Fangshan & Da'anshan & Fangshan & Da'anshan & Fangshan & Da'anshan & Fangshan \\
\hline 233 & Yanchi & Mentougou & Yanchi & Mentougou & Yanchi & Mentougou & Yanchi & Mentougou \\
\hline 234 & Shijiaying & Fangshan & Shijiaying & Fangshan & Shijiaying & Fangshan & Shidu & Fangshan \\
\hline 235 & Shidu & Fangshan & Shidu & Fangshan & Shidu & Fangshan & Shijiayin & Fangshan \\
\hline 236 & Xiayunling & Fangshan & Xiayunling & Fangshan & Xiayunling & Fangshan & Xiayunling & Fangshan \\
\hline 237 & Qingshui & Mentougou & Qingshui & Mentougou & Qingshui & Mentougou & Qingshui & Mentougou \\
\hline 238 & Zhaitang & Mentougou & Zhaitang & Mentougou & Zhaitang & Mentougou & Zhaitang & Mentougou \\
\hline 239 & Puwa & Fangshan & Puwa & Fangshan & Puwa & Fangshan & Puwa & Fangshan \\
\hline
\end{tabular}

accessibility towns with respect to the various economic activities is basically the same as was previously the case with all towns being located in the Fangshan and Mentougou districts. In addition, Haidian District has a particular advantage with respect to educational accessibility, while Dongcheng District needs to further improve its educational accessibility.

3.2. Comprehensive Accessibility to Different Types of Economic Activity. Considering the economic implications of the data, the accessibility to employment, education, medical care, and shopping and leisure were weighted equally, that is, the weight value for each type of accessibility was 0.25 . On this basis, the comprehensive accessibility of 239 accommodation blocks in the study area was calculated and the scores were then classed into five grades (Figure 3) to further analyze the comprehensive distribution patterns for the accessibility of economic activities in Beijing. As shown in Figure 3, the comprehensive accessibility of the selected economic activities in Beijing still exhibits a circular-type structure that decreases in going from the center to the outer suburbs. There were 56 towns with the highest comprehensive accessibility score (score $>130$ ), mostly in the Xicheng and Haidian districts which are within the $4^{\text {th }}$ Ring Road. The status of public transport infrastructure in this region is well developed and mature, and there is a high distribution density of economic activity; therefore, it is very convenient to access the diverse resources and service activities. There were 59 towns with relatively high comprehensive accessibility (score 120-130), most of which are distributed between the $4^{\text {th }}$ and $5^{\text {th }}$ Ring Roads, as well as some areas north of the $5^{\text {th }}$ Ring Road. These areas are close

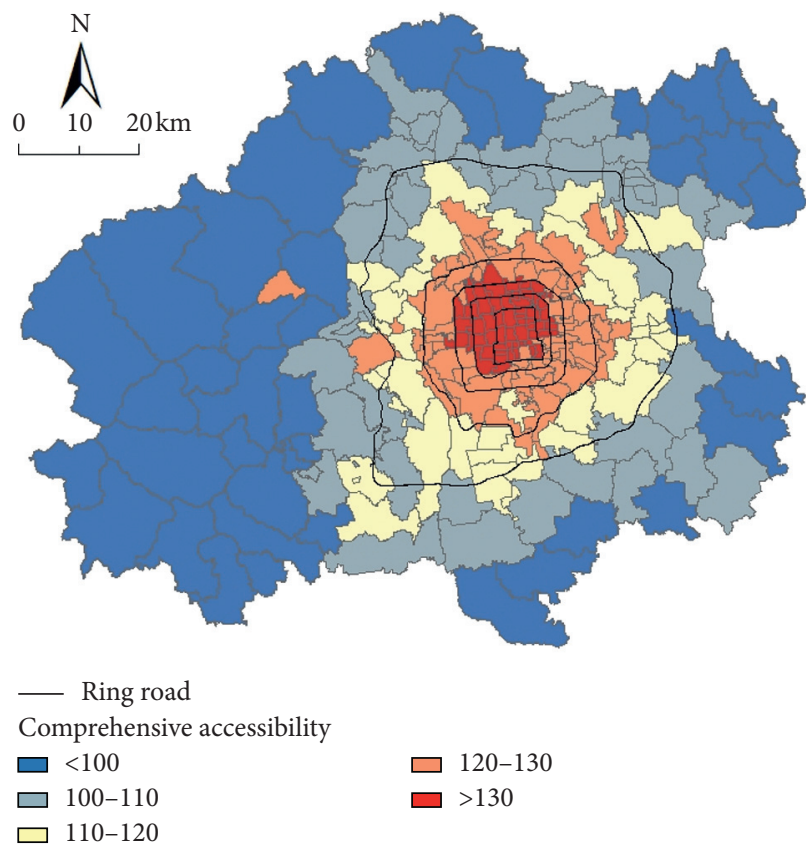

FIgURE 3: Comprehensive accessibility of the study area.

to the central area where the traffic flow is relatively smooth; thus, these locations have the advantage of easy access to services and resources. There were 44 towns with moderate comprehensive accessibility (score 110-120) and these towns are located between the $5^{\text {th }}$ and the $6^{\text {th }}$ Ring Road. The ability to access diverse services and resources in this area is considered to be below average, despite the towns' access to urban economic service activities having a moderate accessibility score. 
There were 38 towns with low comprehensive accessibility (score 100-110). These areas are located outside the $6^{\text {th }}$ Ring Road, far removed from the city center. These towns are inconvenient to access due to poor transport infrastructure and fall outside the service scope of most urban economic activities, such that there is poor accessibility. There were 42 towns with the lowest comprehensive accessibility ranking (score $<100$ ), and most of these are in the Fangshan and Mentougou districts. These areas are remote from the urban areas, lack convenient transportation, and the distribution of various economic activities is quite sparse, and the accessibility is judged to be very low.

Next, we used global autocorrelation [39] and local autocorrelation [40] methods to further analyze whether a comprehensive accessibility index gave an indication of significant spatial clustering. The global spatial autocorrelation analysis showed that Moran's I index was 0.52 , the $z$ score was 37.3 , and the $p$ value was less than 0.00001 . Hence, there is a significant positive correlation in the comprehensive accessibility, indicating that, in the whole study area of Beijing, the trend for spatial clustering of similarly accessible towns is clear, confirming significant regional differences for accessing economic activities.

The Global Moran's I Index reveals a global spatial clustering feature with respect to accessibility of urban economic activity, but it can only reflect the spatial clustering pattern of urban functionally active similarity values (positive correlation) or nonsimilarity observation values (negative correlation) between regions [41]. However, Anselin Local Moran's I index and the Local $G$ coefficient can detect whether a town is a high-value cluster or a low-value cluster; thus, the agglomeration pattern and distribution difference of urban spatial accessibility can be more accurately detected. Based on this, the cluster and outlier analysis (Anselin Local Moran's I) tool of ArcGIS was used to conduct local spatial correlation analysis on the comprehensive accessibility of each town, and the results of clustering and outlier analysis are presented in Figure 4.

A "high-high cluster" is mainly concentrated in the urban center within the $5^{\text {th }}$ Ring Road and within the towns themselves; moreover, adjacent towns also have high economic activity accessibility. This "high-high" cluster enjoys favorable geographical conditions, has an established urban infrastructure, a dense distribution of various economic activities, and an almost ideal road and public transport infrastructure. A "low-low cluster" is concentrated in the Fangshan and Mentougou districts in the west of the city, as well as in parts of Shunyi District in the northeast, where the accessibility of the towns to economic activities (including adjacent towns) is relatively low. Most of these areas are newly built residential areas and the public service facilities in the immediate area are insufficient, so the residents have more limited choices with respect to economic activities. A "lowhigh outlier" was detected in Beiqijia in Changping District. The accessibility of this town was found to be low, but the adjacent towns have a high spatial accessibility with respect to economic activities, which is negatively correlated in space. Compared with the "high-high cluster," the "low-low cluster"
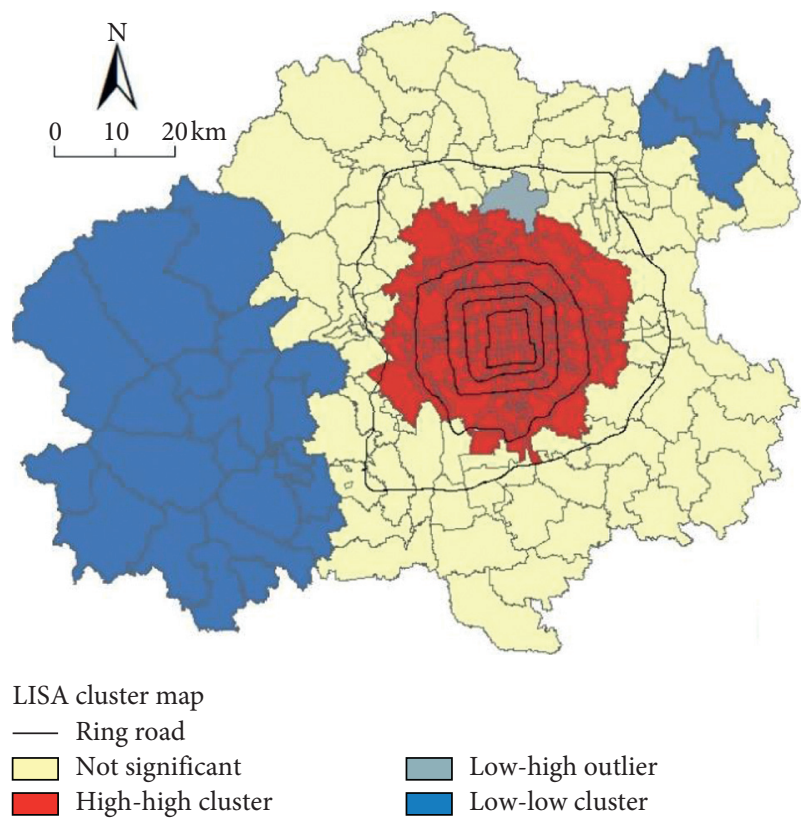

FIGURE 4: LISA cluster map of comprehensive accessibility.

and the "low-high outlier" are generally distributed in the peripheral regions of the city. The low density of economic activities and the limited range of services are the most important factors affecting the residents' access to various economic opportunities. Therefore, in these areas, it is necessary to provide new opportunities that meet the employment, educational, medical care, shopping, and leisure needs of the residents, which, in turn, will stimulate economic activity to improve the spatial accessibility of the area.

\section{Equality Evaluation of Residents' Economic Activity-Related Journeys}

4.1. Distribution of Residents. The accessibility of economic activities is mainly a measure of the ease of obtaining economic activity opportunities within a certain distance from the supply source. The equality evaluation of the opportunities for economic activity-related journeys also needs to consider the requirements of the residents. In previous studies, the demand for economic activities was often characterized by the number of people living in a particular area [42]. In general, an area with a large population has a high demand for all kinds of economic activities. Based on the population statistics of the sixth National Census in 2010, the population distribution of Beijing (Figure 5) was examined. It can be seen from inspection of Figure 5 that the population distribution in the study area is highest in Dongxiaokou, Huilongguan, and Beijiazhen in Changping District, and Xueyuanlu in Haidian District; it is in these towns where there is the greatest demand for economic activities. The populations in the districts of Fangshan, Mentougou, and Shunyi, which are outwith the urban area, are more sparsely distributed, and the demands for economic activities are correspondingly less. 


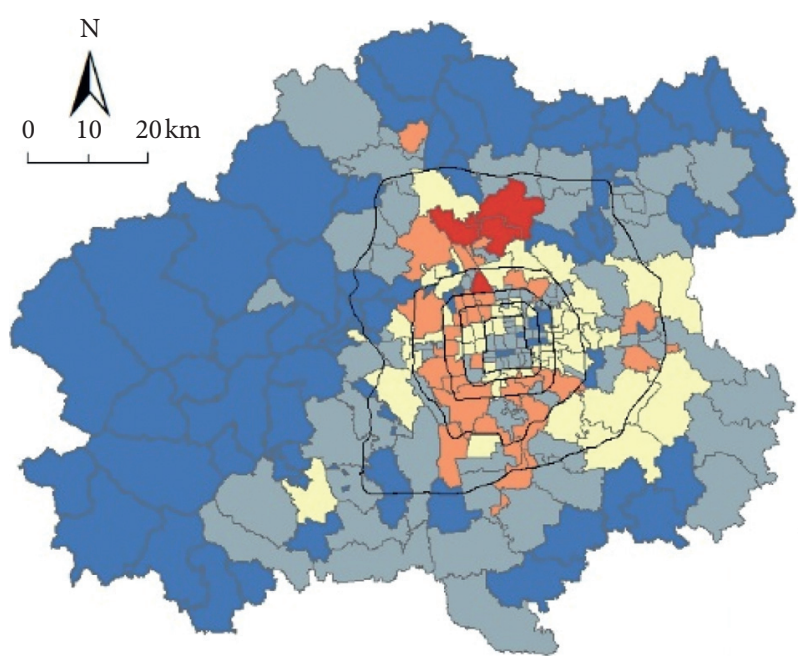

_ Ring road
Population
$\square$ 2472-37959
$\square 37960-75736$
$\square 75737-130925$

FIgUre 5: Population distribution map of the study area.

4.2. Equality Evaluation by Urban Economic Activity. To further explore the relationship between urban accessibility and population distribution, we calculated the population distribution under various economic activity accessibility intervals (Figure 6) and then analyzed the impact of the different economic activities on the residents' choice of housing, in order to reveal the spatial equality of the residents' access to economic activities. As can be seen from Figure 6, the distribution of residents for a particular accessibility interval for the various economic activities has similar characteristics; with increase in accessibility, there is an upward trend in the population agglomeration which generally declines after reaching a maximum level. This indicates that the population generally tends to live in areas with high accessibility; however, the attractiveness of accessibility to the population is eventually limited. Affected by traffic congestion, costs of housing, and other factors, there is a tendency for residents to migrate from urban centers to the peripheral regions in pursuit of a lower cost of living and a better quality of life. Therefore, although the urban center enjoys the highest levels of accessibility, it is still unable to secure the largest numbers of people; thus, a decrease in population occurs for the highest levels of accessibility (Figure 6(a)-6(d)). The residents' requirement for timely and convenient commuting is of the highest importance. About $64.5 \%$ of the population in the study area live in areas with high employment accessibility (score $>120$ ); hence, the employees can benefit from having a convenient commuting experience. Also of importance to residents is the shopping and leisure experience, with about $64.3 \%$ of the population living in towns with a high access to shopping and leisure. Of less importance are medical-related journeys where the figure accounts for $61.6 \%$ of the population. These results show that the choice of location for residents is mainly dictated by the requirements for commuting to work and shopping and leisure travel, and choice is less affected by the need to access medical facilities given that the frequency of such trips is much less than for the former journeys.

Specifically, due to the different locations, the resident populations have different degrees of economic activityrelated opportunities. As far as employment accessibility is concerned, $32.2 \%$ of the population is concentrated in the towns which have the highest employment accessibility (score $>130$ ) and these locations enjoy the best commuting situation. Some $18.7 \%$ of the population lives in towns with an accessibility score of less than 110 , where not only the ability to access employment opportunities is low but also the travel experience to work is also inconvenient. With respect to shopping and leisure accessibility, $29.6 \%$ of the population are distributed in towns with the highest medical accessibility (score of $>130$ ), while more people $(34.8 \%$ ) are distributed in towns with medium accessibility (score 120-130) indicating moderate access to shopping and leisure resources. Besides, $19.2 \%$ of the population live in towns where the shopping and leisure accessibility is low (score $<110$ ); hence, the inhabitants cannot experience a convenient shopping and leisure trip. As for education and medical-related accessibility, only $25.3 \%$ and $22.8 \%$ of the population can access the best journey conditions for education and medical activity-related travel, respectively, while much of the population $(37.12 \%$ and $38.83 \%$, respectively) live in towns with an accessibility score of $120-130$. Furthermore, $17.7 \%$ and $19.5 \%$ of the population live in towns with education and medical activity-related accessibility scores below 110, respectively. These people cannot obtain equal accessibility with respect to undertaking education and medical activity-related journeys. Clearly, the allocation of education and medical resources in these areas needs to be upgraded.

\subsection{Equality Evaluation of the Residents' Economic Activity-} Related Journeys. The distribution data for the economicrelated journeys of the residents at different levels of comprehensive accessibility were calculated and the results are presented in Table 2. Then, combining the population distribution data and the comprehensive accessibility data (Figures 5 and 3), we further analyzed the spatial equality of the supply and demand of comprehensive accessibility. It can be seen that the advantages of the comprehensive accessibility of economic activities in the study area are not entirely consistent with the distribution data for the residents, and there are different types of inequalities.

The area with the highest comprehensive accessibility (score $>130$ ) is located in the central urban area within the 4th Ring Road; this area has a total population of 4.73 million people. Among them, the districts of Xicheng, Dongcheng, and Haidian have the most favorable accessibility situations, and $100 \%, 79.6 \%$, and $57.6 \%$ of the residents in these areas, respectively, experience the best opportunities for undertaking economic activity-related journeys in the city. However, due to the high house prices and living costs in the city center, this population only accounts for $27.8 \%$ of 


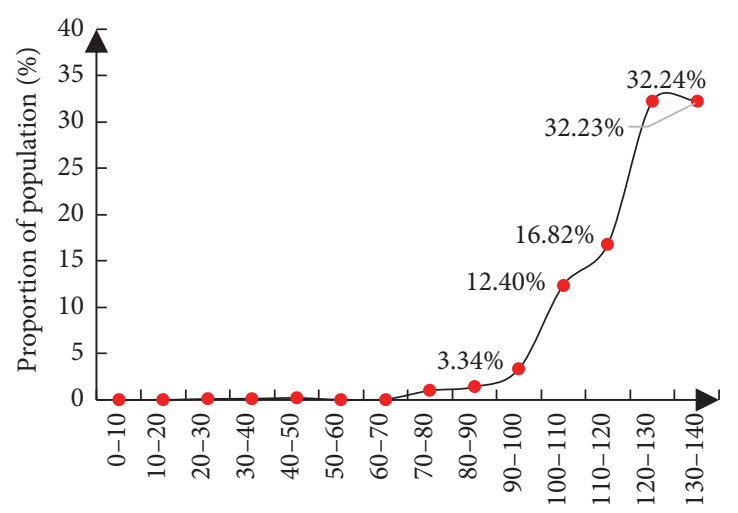

(a)

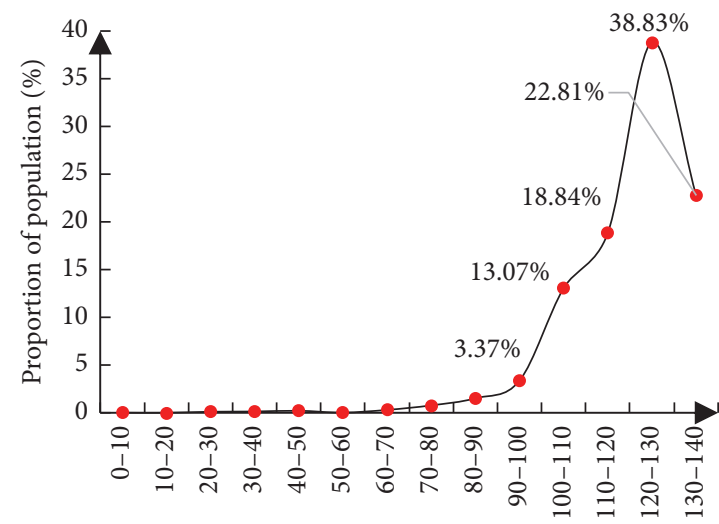

(c)

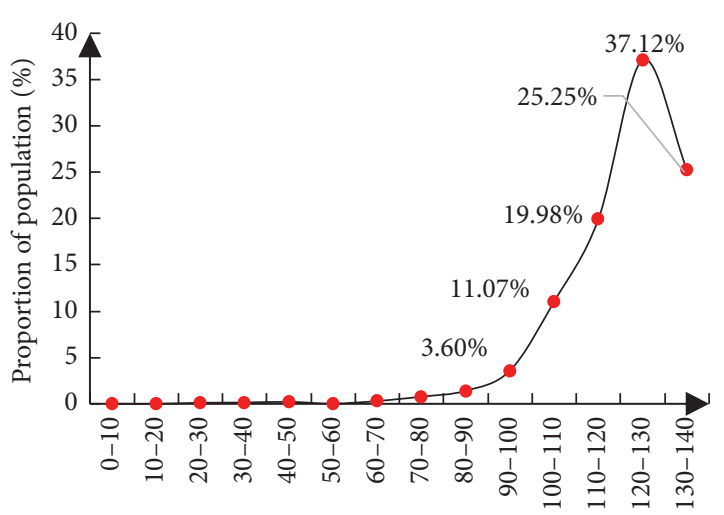

(b)

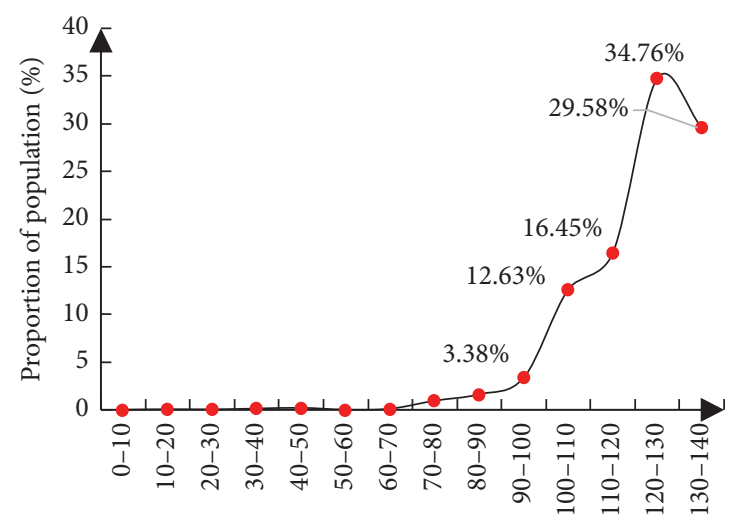

(d)

FIGURE 6: Number of residents for the different economic activities. (a) Employment accessibility. (b) Educational accessibility. (c) Medical accessibility. (d) Shopping and leisure accessibility.

TABle 2: Distributions for residents at different levels of comprehensive accessibility.

\begin{tabular}{|c|c|c|c|c|c|c|}
\hline \multirow{2}{*}{ District } & \multicolumn{5}{|c|}{ Accessibility level } & \multirow{2}{*}{ Total number $(10,000)$} \\
\hline & $<100$ & $100-110$ & $110-120$ & $120-130$ & $>130$ & \\
\hline Xicheng & 0 & 0 & 0 & 0 & 123.28 & 123.28 \\
\hline Dongcheng & 0 & 0 & 0 & 18.78 & 73.15 & 91.93 \\
\hline Haidian & 0 & 14.24 & 17.12 & 107.78 & 188.92 & 328.07 \\
\hline Fengtai & 0 & 5.38 & 16.14 & 124.45 & 14.75 & 160.73 \\
\hline Chaoyang & 0 & 0 & 29.63 & 245.53 & 72.95 & 348.11 \\
\hline Shijingshan & 0 & 0 & 20.94 & 38.38 & 0 & 59.32 \\
\hline Daxing & 15.34 & 22.40 & 55.73 & 33.49 & 0 & 126.96 \\
\hline Tongzhou & 17.73 & 36.79 & 63.91 & 0 & 0 & 118.43 \\
\hline Changping & 15.52 & 72.88 & 47.02 & 30.63 & 0 & 166.05 \\
\hline Mentougou & 5.24 & 10.27 & 9.29 & 4.24 & 0 & 29.05 \\
\hline Shunyi & 27.57 & 34.59 & 11.06 & 3.33 & 0 & 76.55 \\
\hline Fangshan & 28.67 & 25.99 & 20.12 & 0 & 0 & 74.78 \\
\hline Total & 110.06 & 222.54 & 290.97 & 606.63 & 473.05 & 1703.24 \\
\hline
\end{tabular}

the total population. Therefore, only a relatively small number of people benefit from the best transport accessibility opportunities in the city; this situation results in an excessive concentration of economic activities in the urban center and equates to an inequitable distribution of social resources. Clearly, this is not conducive to securing spatial equality for the residents' journeys.

The areas with medium comprehensive accessibility (score 110-130) are mainly located between the 4th and the 6th Ring Road, having more than half of the city's population $(52.7 \%)$. Most of the residents in the Fengtai (97.5\%), Chaoyang (79.0\%), Shijingshan (100\%), Daxing (70.3\%), and Tongzhou districts $(54.0 \%)$ fall into this category. These residents experience medium-level accessibility conditions for economic activity-related journeys, but the supply of and the demand for economic activities are not fully met. The distribution of economic activities such as employment, education, medical care, and transportation cannot match 
the needs of the large population. The limited resources in this area result in inequalities.

The area with poor comprehensive accessibility (score $<110$ ) has a population of 3.33 million and accounts for $19.5 \%$ of the total population. This area is mainly in the suburbs outside the 6th Ring Road and comprises the districts of Changping, Mentougou, Shunyi, and Fangshan. Compared with other regions, the residents in these regions have limited access to economic activity-related resources and there is a lack of public transport; thus, there is limited scope for undertaking journeys; hence, the spatial inequalities between these districts and the more centralized regions. Therefore, it is necessary to develop more equitable plans for all kinds of economic activities throughout Beijing and redress the imbalance of the transportation infrastructure in marginal areas, so as to ensure more equitable transport opportunities for residents in the whole city.

\section{Discussion}

Research concerning the accessibility and equality of urban economic activities can provide important information for urban planning including resource allocation. Such work helps in the planning and optimization of future urban economic activities and transport infrastructure and facilitates the fair distribution of economic-related opportunities. In the traditional approach to evaluating urban services accessibility, the types of economic activities are rarely distinguished. However, diversification of economic activities is of great significance to land use development and adjustment of the urban spatial structure. Therefore, this study has focused on the spatial differences of accessibility based on four types of economic activity. The accessibility model established for a comprehensive selection of economic activities makes up for the deficiencies of previous accessibility evaluations and emphasizes the access opportunities to diverse economic activities for different towns; in effect, this model serves as an important indicator for revealing the equality of the residents' economic activity-related journeys. In addition, in a previous equality evaluation of residents' journeys, the impact of the participants' needs on the evaluation results was rarely considered, and only the fairness of the distribution of economic activity-related facilities from a geographic perspective was measured. With regard to the balance between supply and demand for urban services, this study makes up for the shortage of a space equality evaluation method that is normally undertaken from a single viewpoint and proposes a more comprehensive approach for evaluation of the equality of economic activityrelated journeys undertaken by Beijing's residents.

Several recommendations can be proposed based on the results of this study. When considering future economic activities, urban planners and decision makers should adopt an evaluation method which examines the spatial accessibility of urban economic activities, consider comprehensively the spatial distribution of the residents, and take into consideration the residents' commuting and other economic activity-related travel factors including, for instance, the allocation of resources for economic activities and selection of routes and stations within the urban rail transit system. Also, from the perspective of the spatial equality of opportunities, on the one hand, the government should establish appropriate educational, medical, shopping, and leisure facilities for the suburbs and towns which are well removed from the center of Beijing and pay more attention to the construction of transportation infrastructure at the peripheries of urban areas. On the other hand, the overconcentrated economic service functions in the center of the city should be decentralized to improve and realize a more equitable transport system.

However, there are still some limitations to the study in terms of spatial equality analysis. Due to the limitations of the research data, there is mismatch between the dates of the economic activity data and the demographic data; this needs to be addressed; however, the present findings are considered robust and adequate for the purposes of explaining the spatial equality problem. This research has focused on the study of the equality of economic activity-related journeys made by the residents of Beijing, but it does not take into account the demographic structures and special social groups (such as children, the elderly, and groups of different races) and other factors. In future research, the personalized needs of different populations in the context of economic activities need to be further considered, so as to further improve the accessibility model and spatial equality evaluation.

\section{Conclusions}

In this paper, the spatial distribution characteristics and the spatial equality of economic activity-related accessibility in Beijing were analyzed. First, based on the distribution of urban economic activities and the transportation systems, an accessibility model was built to calculate the accessibility of four major economic activities, namely, employment, education, medical care, and shopping and leisure. The model enabled us to analyze the respective spatial distribution accessibility characteristics associated with the four types of economic activity. Second, we weighted the four types of accessibility equally to obtain a comprehensive accessibility index and analyzed the spatial agglomeration pattern using a global autocorrelation analysis method. Finally, the spatial equalities of the economic activities were explained by factoring in the spatial distribution of the resident population in Beijing. The main conclusions are as follows:

(1) The accessibility of various economic activities in Beijing generally follows a law whereby accessibility decreases from the city center to the peripheral suburbs, and a "core to edge" feature is clear. The area within the $4^{\text {th }}$ Ring Road represents the location with strong accessibility for employment, and Haidian District has a clear advantage in terms of education accessibility. The accessibility of medical services and shopping and leisure has similar distributions with concentrations in the main urban area within the $5^{\text {th }}$ Ring Road. More specifically, the towns with high accessibility to all kinds of economic activities are mostly distributed in Xicheng 
District and include Jinrongjie, Yuetan, Xinjiekou, and Shichahai.

(2) The districts with the highest level of comprehensive accessibility are Xicheng District and Haidian District, while Fangshan District and Mentougou District have the lowest level of comprehensive accessibility. The trend towards the spatial agglomeration of towns with similar accessibility levels is clear and results in significant regional differences with respect to access to economic activities. The urban center within the $5^{\text {th }}$ Ring Road enjoys a high degree of accessibility and superior infrastructure conditions. In the districts of Fangshan, Mentougou, and Shunyi, there are some areas where the towns with low levels of accessibility group together; overall the infrastructure conditions are poor in these locations.

(3) In general, towns with large populations have a high demand for all kinds of economic activities. Dongxiaokou, Huilongguan, and Beijiazhen in Changping District and Xueyuanlu in Haidian District have the largest demand for economic activities, whereas there is much less demand in the districts of Mentougou, Fangshan, and Shunyi. The graph of the relationship between the accessibility level and the number of residents shows that the population generally tends to settle in the areas with high accessibility, but the attractiveness of accessibility has an upper limit, there being a reduction in population for the central urban area at the highest levels of accessibility. In terms of the attractiveness of economic activities for residents, the need for transportation to the workplace is the most important factor, while the need to access medical facilities is the least important of the four economic categories examined.

(4) The advantageous locations regarding comprehensive accessibility of economic activities and distribution of residents are not entirely consistent. Some $27.8 \%$ of the population in the study area live in the region with the highest comprehensive accessibility (score $>130$ ), which is mainly in the districts of Xicheng, Dongcheng, and Haidian, these districts having the best opportunities for economic activityrelated journeys. More than half of the population (52.7\%) live in areas with moderate comprehensive accessibility (score 110-130); these areas include the districts of Fengtai, Chaoyang, Shijingshan, Daxing, and Tongzhou. The distribution of economic activities and traffic conditions in these areas cannot meet the needs of a large number of people, and this results in inequalities due to a relative scarcity of resources. Some $19.5 \%$ of the population live in areas with a low comprehensive accessibility score (score $<110$ ), mostly in the Changping, Mentougou, Shunyi, and Fangshan districts. Residents in these areas have poor economic activity-related journey opportunities, and, moreover, journeys made by residents between these regions are not equal in terms of accessibility.

\section{Data Availability}

The economic activity distribution data and demographic data used to support the findings of this study were supplied by National Bureau of Statistics of China under license and so cannot be made freely available. Requests for access to these data should be made to Yang, (yangxy.19s@ igsnrr.ac.cn).

\section{Conflicts of Interest}

The authors declare that they have no conflicts of interest.

\section{Acknowledgments}

This study was supported by the National Natural Science Foundation of China (Grant No. 42071153), Strategic Priority Research Program of the Chinese Academy of Sciences (Grant No. XDA20080000).

\section{References}

[1] K. Y. Wang and L. Xiao, "Analysis on the motive mechanism of developing for urban spatial structure," Journal of South China Normal University (Natural Science Edition), vol. 3, pp. 116-122, 2005.

[2] Y. Wang, Analysis of Mechanism and Routes of Regional Urban Space Restructuring in Northeast China, Northeast Normal University, Changchun, China, 2012.

[3] B. Cohen, "Urbanization in developing countries: current trends, future projections, and key challenges for sustainability," Technology in Society, vol. 28, no. 1-2, pp. 63-80, 2006.

[4] K. W. Tsou, Y. T. Hung, and Y. L. Chang, "An accessibilitybased integrated measure of relative spatial equity in urban public facilities," Cities, vol. 22, no. 6, pp. 424-435, 2005.

[5] E. Talen and L. Anselin, "Assessing spatial equity: an evaluation of measures of accessibility to public playgrounds," Environment and Planning A: Economy and Space, vol. 30, no. 4, pp. 595-613, 1998.

[6] E. Talen, "The social equity of urban service distribution: an exploration of park access in Pueblo, Colorado, and Macon, Georgia," Urban Geography, vol. 18, no. 6, pp. 521-541, 1997.

[7] Q. X. Chen, Y. Hou, and S. Wu, "Assessment of accessibility to urban parks in Shaoxing City from the perspective of opportunity equity," Scientia Geographica Sinica, vol. 36, pp. 375-383, 2016.

[8] Z. B. Liu, C. Zhang, and T. Q. Dai, "Measuring accessibility of multi-type urban public service facilities with entropy in Beijing," Economic Geography, vol. 38, pp. 77-84, 2018.

[9] W. G. Hansen, "How accessibility shapes land use," Journal of the American Institute of Planners, vol. 25, no. 2, pp. 73-76, 1959.

[10] G. H. Pirie, "Measuring accessibility: a review and proposal," Environment and Planning A: Economy and Space, vol. 11, no. 3, pp. 299-312, 1979.

[11] J. A. Pooler, "The use of spatial separation in the measurement of transportation accessibility," Transportation Research, Part A (Policy and Practice), vol. 29, pp. 1-427, 1995. 
[12] D. Halden, P. Jones, and S. Wixey, Accessibility Analysis Literature Review: Measuring Accessibility as Experienced by Different Socially Disadvantage Groups, pp. 1-55, 2005.

[13] L. A. Aday and R. Andersen, "A framework for the study of access to medical care," Health Services Research, vol. 9, pp. 208-220, 1974.

[14] H. W. Yin and F. H. Kong, "Accessibility analysis of urban green space in Jinan," Journal of Plant Ecology, vol. 30, pp. 17-24, 2006.

[15] S. Kalogirou, R. Foley, P. Health, and Hanly, "Health, place and Hanly: modelling accessibility to hospitals in Ireland," Irish Geography, vol. 39, no. 1, pp. 52-68, 2006.

[16] T. S. Hare and H. R. Barcus, "Geographical accessibility and Kentucky's heart-related hospital services," Applied Geography, vol. 27, pp. 1-205, 2007.

[17] L. Rosero-Bixby, "Spatial access to health care in costa rica and its equity: a GIS-based study," Social Science \& Medicine, vol. 58, no. 7, pp. 1271-1284, 2004.

[18] Y. Liu, Y. Jing, L. Zhang et al., "Assessing spatial accessibility of public and private residential aged care facilities: a case study in Wuhan, central China," ISPRS International Journal of Geo-Information, vol. 6, p. 304, 2017.

[19] Y. Gao, Q. He, Y. Liu, L. Zhang, H. Wang, and E. Cai, "Imbalance in spatial accessibility to primary and secondary schools in China: guidance for education sustainability," Sustainability, vol. 8, no. 12, p. 1236, 2016.

[20] Y. H. Han and Y. Q. Lu, "Accessibility assessment and planning of public service facilities for education: a case study on senior high schools in Yizheng City," Geographica Sinica, vol. 32, pp. 822-827, 2012.

[21] J. P. Sung, "Measuring public library accessibility: a case study using GIS," Library \& Information Science Research, vol. 34, pp. 13-21, 2012.

[22] M.-P. Kwan, A. T. Murray, M. E. O’Kelly, and M. Tiefelsdorf, "Recent advances in accessibility research: representation, methodology and applications," Journal of Geographical Systems, vol. 5, no. 1, pp. 129-138, 2003.

[23] S. L. Handy and D. A. Niemeier, "Measuring accessibility: an exploration of issues and alternatives," Environment and Planning A: Economy and Space, vol. 29, no. 7, pp. 1175-1194, 1997.

[24] M. D. Gu and H. W. Yin, "Research review on the spatial accessibility and equity of public facility," Urban Problems, vol. 5, pp. 25-29, 2010.

[25] J. S. Wu, M. L. Si, and W. F. Li, "Spatial equity analysis of urban green space from the perspective of balance between supply and demand: a case study of Futian district, Shenzhen,China," Chinese Journal of Applied Ecology, vol. 27, pp. 2831-2838, 2016.

[26] L. A. Guzman, D. Oviedo, and C. Rivera, "Assessing equity in transport accessibility to work and study: the Bogotá region," Journal of Transport Geography, vol. 58, pp. 236-246, 2017.

[27] B. Wail, S. Hanchane, and A. Kamal, "A new data set of educational inequality in the world, 1950-2010: gini index of education by age group," SSRN Electronic Journal, vol. 104, pp. 184-198, 2011.

[28] O. A. Ajala and K. Asres, "Accessibility in equality to basic education in amhara region, Ethiopia," Ethiopian Journal of Education \& Sciences, vol. 3, pp. 11-26, 2008.

[29] W. Pan, H. Fu, and P. Zheng, "Regional poverty and inequality in the xiamen-zhangzhou-quanzhou city cluster in China based on NPP/VIIRS night-time light imagery," Sustainability, vol. 12, pp. 2-20, 2020.
[30] P. Townsend, Poverty in United Kingdom: A Survey of Household Resources and Standards of Living, pp. 15-50, Penguin, London, UK, 1979.

[31] N. Heynen, H. A. Perkins, and P. Roy, "The political ecology of uneven urban green space," Urban Affairs Review, vol. 42, no. 1, pp. 3-25, 2006.

[32] J. B. Gao, C. S. Zhou, Y. M. Wang et al., "Spatial analysis on urban public service facilities of Guangzhou City during the economy system transformation," Geographical Research, vol. 30, pp. 424-436, 2011.

[33] C. Yin, Q. He, Y. Liu, W. Chen, and Y. Gao, "Inequality of public health and its role in spatial accessibility to medical facilities in China," Applied Geography, vol. 92, pp. 50-62, 2018.

[34] Y. D. Wei and C. C. Fan, "Regional inequality in China: a case study of Jiangsu Province," The Professional Geographer, vol. 3, pp. 455-469, 2010.

[35] Y. D. Wei, "Spatiality of regional inequality," Applied Geography, vol. 61, pp. 1-10, 2015.

[36] L. Hu, T. Sun, and L. Wang, "Evolving urban spatial structure and commuting patterns: a case study of Beijing, China," Transportation Research Part D: Transport and Environment, vol. 59, pp. 11-22, 2018.

[37] F. Q. Niu, W. D. Liu, and J. X. Feng, "Modeling urban housing price: the perspective of household activity demand," Acta Geographica Sinica, vol. 71, pp. 1731-1740, 2016.

[38] D. McFadden, "Modelling the choice of residential location," in Spatial Interaction Theory and Planning Models, A. Karlqvist et al., Ed., pp. 75-96, North Holland, Amsterdam, Netherland, 1978.

[39] A. D. Cliff, Spatial Autocorrelation, pp. 7-17, Pion, London, UK, 1973.

[40] R. Kosfeld, H.-F. Eckey, and M. Türck, "LISA (local indicators of spatial association)," WiSt-Wirtschaftswissenschaftliches Studium, vol. 36, no. 3, pp. 157-162, 2007.

[41] S. P. Chen, M. Fang, and D. Zhuang, "Analysis of road density spatial heterogeneity and its influences for urban form of Guangzhou," Geomatics World, vol. 26, pp. 37-43, 2019.

[42] I. Omer, "Evaluating accessibility using house-level data: a spatial equity perspective," Computers, Environment and Urban Systems, vol. 30, no. 3, pp. 254-274, 2006. 